\title{
DeepSF: Deep Convolutional Neural Network for Mapping Protein Sequences to Folds
}

\author{
Jie Hou \\ Department of Electrical Engineering \\ and Computer Science \\ University of Missouri, Columbia, MO \\ USA \\ jh7x3@mail.missouri.edu
}

\author{
Badri Adhikari \\ Department of Computer Science \\ University of Missouri, St. Louis, MO \\ USA \\ adhikarib@umsl.edu
}

\author{
Jianlin Cheng \\ Department of Electrical Engineering \\ and Computer Science \\ University of Missouri, Columbia, MO \\ USA \\ chengji@missouri.edu
}

\begin{abstract}
Predicting the fold of a protein from its sequence is a challenging and important problem in bioinformatics. In this work, we developed the first deep learning method that can directly classify any protein sequence of arbitrary length into 1,195 known protein folds in the Structural Classification of Proteins (SCOP) database [1]. The method uses one-dimensional convolutional neural networks (1D-CNN) to automatically extract features from raw protein sequence information and compose them to high-level features to predict protein folds, achieving a classification accuracy of $73 \%$ on an independent test dataset. This novel machine learning approach is different from traditional alignment methods that rely on the known folds of homologous proteins to recognize the fold of a target protein. It is also more accurate than HHSearch - a top protein profileprofile alignment method in recognizing folds of proteins that have little sequence similarity with proteins with known folds. Moreover, the deep learning method overcomes the shortcoming of the previous machine learning methods that can only classify proteins into dozens of pre-selected folds due to methodological limitations. The hidden features extracted by the deep learning method provide a new semantic representation of proteins that can be used in other protein analysis tasks such as protein clustering and comparison.
\end{abstract}

\section{CCS CONCEPTS}

- Bioinformatics $\rightarrow$ Protein folding •

\section{KEYWORDS}

Protein fold classification, deep learning, convolutional neural network

\section{ACM Reference format:}

J. Hou, B. Adhikari, and J. Cheng. 2018. DeepSF: deep convolutional neural network for mapping protein sequences to folds. In Proceedings of 9th ACM Conf. on Bioinformatics, Computational Biology, \& Health Informatics (ACM-BCB'18), Aug. 29-Sept. 1, 2018, Washington, DC, USA ACM, NY, NY. 1 page. https://doi.org/10.1145/3233547.3233716

\section{ACKNOWLEDGMENTS}

This work was partially supported by two NSF grants (DBI1759934 and IIS1763246) and an NIH grant (R01GM093123).

\section{REFERENCES}

[1] J. Hou, B. Adhikari, J. Cheng. DeepSF: deep convolutional neural network for mapping protein sequences to folds. Bioinformatics 34, 8 (2018), 1295-1303.
Permission to make digital or hard copies of part or all of this work for personal or classroom use is granted without fee provided that copies are not made or distributed for profit or commercial advantage and that copies bear this notice and the full citation on the first page. Copyrights for third-party components of this work must be honored. For all other uses, contact the owner/author(s).

ACM-BCB'18, August 29-September 1, 2018, Washington, DC, USA

(C) 2018 Copyright is held by the owner/author(s)

ACM ISBN 978-1-4503-5794-4/18/08.

https://doi.org/10.1145/3233547.3233716 